\title{
Managing COVID-19 infection in living donation kidney transplant recipient: a single center experience
}

\author{
Ni Made Hustrini, Anandhara Indriani Khumaedi
}

Department of Internal Medicine, Faculty of Medicine Universitas Indonesia, Central Jakarta, Indonesia

Background: COVID-19 is detrimental for those with comorbidities. Kidney transplant recipients are at the highest risk for developing severe COVID-19 due to their immunocompromised status. We perform a review of COVID-19 infection in our kidney transplant recipients who underwent transplantation from year 2014-2020.

Methods: We conducted a cross-sectional study of 12 kidney transplant recipients who were infected with COVID-19 from January 2020-July 2021. Data was collected through electronic health record. Clinical data includes presenting symptoms, duration from onset to hospitalization, COVID-19 severity, use of mechanical ventilation and any forms of renal replacement therapy, and laboratory values. COVID-19 severity is divided into three categories based on our local guidelines: mild (no evidence of pneumonia), moderate (clinical evidence of pneumonia without dyspnea or supplemental oxygen requirement), and severe-critical (acute respiratory distress syndrome at presentation or severe dyspnea requires supplemental oxygen).

Results: Prevalence of COVID-19 infection in kidney transplant recipient is 0,02\% (12/689). Most of the patients are male (83\%) and $67 \%$ patients were diagnosed with severe-critical COVID-19. Fifty percent of patients (6/12) were died and one is still in hospitalization. Among non-survivor we found a trend towards older age (58 [22.5] vs. 54 [31.5] years; median [interquartile range, IQR]), longer time to seek for medical assistance (6 [10.9] vs. 2 [3] days; median [IQR]), having multiple comorbidities, as well as higher inflammatory markers (C-reactive protein, 156.3 [173.8] vs. 42.9 [97.9] mg/L; D-dimer, 7,590 [5,615] vs. 820 [2,180] $\mu \mathrm{g} / \mathrm{mL}$; median [IQR]). Immunosuppressants were discontinued/adjusted in all moderate-severe cases. None of the patients who required mechanical ventilation $(16 \%, 2 / 12)$ and dialysis $(16 \%, 2 / 12)$ were survived. Ninety-nine percent of non-survivors were not vaccinated/unknown. Meanwhile, one fully vaccinated patient had severe-critical COVID-19 and survived.

Conclusions: Early diagnosis and timely management for COVID-19 infection in kidney transplant recipients are mandatory. Nevertheless, vaccination might have a significant impact in preventing worse outcome of COVID-19 infection in kidney transplant recipients.

Corresponding author: Ni Made Hustrini

E-mail:madekum99@gmail.com

(C) The Korean Society for Transplantation

This is an Open Access article distributed under the terms of the Creative Commons Attribution Non-Commercial License (http://creativecommons.org/licenses/by-nc/4.0/) which permits unrestricted non-commercial use, distribution, and reproduction in any medium, provided the original work is properly cited. 